\title{
Liderazgo en los servicios de salud
}

\section{Leadership in health services}

\author{
Olga Pinzón Espitia ${ }^{1}$
}

\begin{abstract}
RESUMEN
El liderazgo en los servicios de salud, implica comprender el primero como un componente fundamental del proceso de gestión organizacional, como competencia de los trabajadores para impactar la satisfacción laboral y la calidad de la atención. El objetivo de este estudio fue revisar algunos tópicos investigados en los últimos años acerca del liderazgo en los servicios de salud.

La importancia de estudiar el efecto transformador del liderazgo a nivel de las instituciones puede contribuir a la gestión exitosa y a la satisfacción misma de los trabajadores, conllevando a plantear la necesidad de consolidar programas de formación en habilidades y competencias de liderazgo para favorecer mejores resultados en las instituciones de servicios de salud. Las ventajas relativas del liderazgo como un proceso en las organizaciones conlleva al empoderamiento de equipos, lo cual se podrá ver reflejado en el rendimiento mismo de los equipos.
\end{abstract}

Es fundamental el papel que desempeñan los departamentos de educación de los hospitales los cuales al articularse con la dirección estratégica de las organizaciones, en pro de la gestión y la eficacia de los procesos, pueden generar programas de formación en habilidades de liderazgo para favorecer mejores resultados institucionales.

Palabras Claves: Liderazgo, Servicios de Salud, Sector Salud, Satisfacción laboral.

1 Doctorando en Ciencias de la Dirección, Universidad del Rosario. Jefe de Servicio, Méderi. Correspondencia: Universidad del Rosario, Sede Complementaria (Norte) _ Módulo D. luciapinzon@yahoo.com 
Leadership in health services, means understanding leadership as a key component of the process of organizational management, as competition for workers to impact job satisfaction and quality of care. The aim of this study was to review some topics investigated in recent years about leadership in health services.

The importance of studying the transformative effect of leadership-level institutions can contribute to the successful management and the same worker satisfaction, leading to raise the need to strengthen training programs in leadership skills and competencies to promote better results in health care institutions. The relative advantages of leadership as a process in organizations leads to the empowerment of teams, which may be reflected in the performance of the equipment itself.

It is essential the role of education departments of hospitals with which to articulate the strategic direction of organizations towards the management and effectiveness of processes can generate training programs in leadership skills to promote better institutional.

Keywords: Leadership, Health Services, Health Sector, Job Satisfaction.

\section{INTRODUCCIÓN}

El liderazgo en los servicios en salud, es un concepto que articula la capacidad para el mejoramiento de la prestación del servicio con la comprensión del contexto, involucrando diferentes actores que tienen como fin último favorecer cambios para el beneficio de la población.

En la última década, la evolución de los sistemas de salud ha exigido cambios de paradigmas con el fin de mejorar la eficiencia, eficacia y calidad de la prestación de los servicios. Dicho cambio implica nuevos planteamientos alrededor de la forma como se lideran las instituciones y se dirigen los equipos de salud, estos últimos con grandes 
problemáticas de formación, motivación, reconocimiento y compromiso. Al respecto Sotelo (1), señala que a nivel mundial los recursos humanos se consideran un área crítica, asociado a profesionales poco adiestrados y sobre todo sin motivación, frecuentemente la contratación y el adiestramiento no se encuentran integrados y muestran grandes incoherencias, la causa principal de esta problemática pareciera estar en la práctica de la gestión y en los sistemas de apoyo del personal.

Se está un momento en el que los directivos a cargo de la gestión de los servicios sanitarios, comprendan que liderar el talento humano requiere replantear estilos de gestión y liderazgo (2). Frente a la necesidad de propuestas novedosas, estudiar e identificar los estilos de liderazgo en los servicios de salud como efecto transformador a nivel de la sociedad para la cual prestan los servicios, como para el bienestar mismo de los trabajadores que contribuyen al logro de la misión de las instituciones de salud, puede contribuir a establecer prioridades estratégicas en el sector.

El propósito del presente artículo es revisar algunos tópicos investigados en los últimos años acerca del liderazgo en los servicios de salud, dado el interés actual en las organizaciones en el liderazgo como variable esencial para la gestión de las instituciones del sector y generador de impacto positivo en la dirección de los equipos de salud. Estudiar el liderazgo en los servicios de salud, implica comprender el liderazgo como un componente fundamental del proceso de gestión y el liderazgo como competencia de los trabajadores en los servicios de salud el cual impacta la satisfacción laboral y la calidad de la atención.

La Organización para la Cooperación y el Desarrollo Económico (OECD) desde la perspectiva de la atención en salud de los pacientes afirma que es cada vez más importante trabajar mancomunadamente con el recurso humano en la prestación de servicios con calidad, ya que cada vez más los sistemas de salud se esfuerzan por ser más sensibles a las necesidades de las personas que utilizan sus servicios (3). 


\section{EL LIDERAZGO EN LOS SERVICIOS DE SALUD}

Un estudio realizado en cinco hospitales del noreste de Estados Unidos describe el liderazgo como una variable que afecta la habilidad, el trabajo en equipo y que contribuye al desarrollo organizacional (4). Así mismo se han estudiado el rol de los líderes en la atención en salud, en cuanto a la contribución de la concepción positiva de la organización, planteando cinco temas claves, como son visión futura, capacidad de identificar y utilizar las habilidades complementarias de los demás y compromiso con el aprendizaje (5).

Por estas razones, el liderazgo en el sector salud se está constituyendo en un factor que contribuye a la prestación de servicios con altos estándares de calidad, como prioridad internacional para todas las instituciones prestadoras de servicios de salud. Diversos estudios e informes ponen en evidencia lo anterior, por ejemplo un artículo referente al sistema de salud, hace referencia a la identificación de cinco competencias de liderazgo necesarias para los profesionales de enfermería que se encuentran en altos cargos directivos, estos son el conocimiento de sí mismo, la visión estratégica, la toma de riesgos y creatividad, la comunicación interpersonal eficiente y ser un líder inspirador de cambio $(6,7)$.

Utilizando como referente el liderazgo y los servicios de salud, desde la perspectiva de análisis es necesario abordar el liderazgo desde las investigaciones realizadas en torno a la gestión de las instituciones y el liderazgo como competencia de los trabajadores en los servicios de salud.

\section{EL LIDERAZGO EN LA GESTIÓN DE INSTITUCIONES DE SERVICIOS DE SALUD}

Aunque desde hace varias décadas se viene realizando investigación en el tema de liderazgo como factor fundamental para una dirección exitosa, es un tema de interés empresarial desde la perspectiva de los altos cargos directivos y gerenciales y el personal que lideran; un ejemplo de ello son las investigaciones en liderazgo, eficiencia $y$ efectividad en los servicios de salud para comprender la gestión de liderazgo para el cambio como factor importante para superar la resistencia al cambio y mejorar las relaciones laborales en un entorno que es cada vez más difícil, y que requiere de diferentes enfoques para la comprensión de liderazgo y la gestión los cuales se articulan 
junto con otras estrategias de liderazgo para mejorar la efectividad de los líderes en el marco del desempeño organizacional (8). Lo anterior guarda relación, con el liderazgo como un requisito fundamental para el éxito de cualquier organización. Centrándose principalmente en la gestión del cuidado del paciente, la mayoría de las organizaciones de atención en salud no tienen programas de desarrollo de liderazgo como componente de la formación y gestión del recurso humano, desafiando los resultados de la gestión, unido a una fuerza laboral que envejece, a la escasez de enfermeras, y los rápidos cambios en los sistemas de salud, por lo que la formación en liderazgo es una necesidad en la gestión de las instituciones que puede contribuir a la retención y formación del personal de atención de salud (9) .

En este sentido, gestionar una institución en el sector salud, implica gestionar el liderazgo y el personal en pro de los mejores resultados, revisando periódicamente su organización y sus estructuras de gestión para responder a los nuevos desarrollos y desafíos del sector con el fin de alcanzar y mantener un alto nivel de eficiencia en la prestación de la asistencia sanitaria (10).

Otro aspecto fundamental en la dirección de las instituciones de servicios de salud, es la contribución principal de un modelo de liderazgo para el cambio, con el cual se puede demostrar ampliamente como el liderazgo es un facilitador efectivo para el mejoramiento de los servicios en una variedad de contextos del sector salud, y a pesar de las condiciones adversas de los sistemas de aseguramiento, el cual en todos los casos de cambios en la gestión, el liderazgo produce la mejora positiva del servicio, hallazgo que es común en las investigaciones realizadas en diversos escenarios organizacionales (1113).

Un buen liderazgo es eficaz para la gestión y juega un papel fundamental en el logro de un cambio exitoso en una cultura de trabajo positiva, lo cual se logra a través de programas innovadores de liderazgo y proyectos de investigación, para favorecer la calidad de los servicios, satisfacción de los beneficiarios de la atención, y reducir los costos asociados(14) .

En la actualidad se puede evidenciar el estudio de importantes procesos de cambios en los servicios de salud y el rol del liderazgo, existen interrogantes acerca de cómo en el 
sector los gerentes y líderes que trabajan en organizaciones tradicionales, racionales y mecanicistas deben tener capacidad y competencias para girar la gestión hacia las capacidades de liderazgo necesarias en las economías emergentes en la gestión de la organizaciones y la prestación de servicios a través de colaboraciones en red y como conectar el liderazgo con el aprendizaje en el trabajo, la educación y el desarrollo profesional en pro de la innovación en la gestión del servicio (15).

\section{LIDERAZGO Y GESTIÓN DE LOS SERVICIOS DE SALUD}

Al analizar las competencias de liderazgo del recurso humano en salud surge un interrogante referentes a cuál es el papel de los directivos y gerentes en la generación de procesos y formación en liderazgo como componente fundamental de la planeación de la dirección de los servicios de salud, responder esta pregunta implica una revisión de las capacidades de los directores y gerentes para identificar contextos y asumir los cambios. La Academia de Liderazgo del Servicio Nacional de Salud del Reino Unido (NHS), en el marco de las competencias de liderazgo de los clínicos (16), platea que es necesario demostrar conocimiento de la vida política, social, técnico, entorno económico, organizativo y profesional, conocer e interpretar la legislación y la rendición de cuentas correspondiente, anticipar y prepararse para el futuro mediante la exploración de las ideas, el mejoramiento de la práctica y las nuevas tendencias que tendrán un impacto en la salud y los resultados.

La concepción acerca del conocimiento de los líderes en la legislación y la gestión de procesos, favorece los resultados y la calidad de la atención, un estudio describe como durante la última década se han estudiado 115 organizaciones de salud, analizando los procesos de gestión desde las salas de juntas a la cama del paciente, encontrando un elemento crítico de casi todos los procesos, la falta de normas conjuntas que puedan conllevar a la confiabilidad del cuidado en salud con cero defectos para los pacientes; esta falta de normas se debe en gran parte a los estilos de gestión jerárquicos y una falta de estructuración del proceso de liderazgo (17). 


\section{https://doi.org/10.22519/21455333.413 \\ LIDERAZGO COMO COMPETENCIA DE LOS TRABAJADORES Y CONCEPCIÓN \\ DE GÉNERO EN LOS SERVICIOS DE SALUD}

La formación en liderazgo de los trabajadores de la salud es un recurso para las personas que buscan contribuir con los resultados de la gestión de instituciones de servicio de salud y una carrera de éxito, por lo que los programas educativos en liderazgo son una estrategia en la gestión del recurso humano que ayuda tanto a los trabajadores como a las organizaciones a desarrollar modelos de atención que incluyan mejoras eficientes y desarrollo de competencias específicas para la industria de la salud (18).

Asumir la necesidad de procesos de liderazgo en las instituciones de salud, implica que la una responsabilidad de la dirección con el bienestar de los equipos de trabajo; con el objetivo de resolver la ambigüedad respecto a las ventajas relativas del empoderamiento y el liderazgo de equipos, se plantea que la dirección de recursos humanos se centre en la influencia de la formación en liderazgo en el desarrollo del equipo a través del tiempo, los resultados empíricos basados en los datos de rendimiento longitudinales de 60 equipos sugirieron que equipos dirigidos por un directivo líder generan empoderamiento y mayor rendimiento en el tiempo, debido a los altos niveles de aprendizaje que se logran alcanzar (19).

Otro tema de interés en las investigaciones, es la habilidad de los líderes de trabajar en función de la práctica basada en la evidencia (EBP) y la gestión del riesgo, lo cual ha aumentado en la última década. Sin embargo, el número de líderes con habilidades para la toma de riesgos no ha experimentado un crecimiento similar, por lo que a las habilidades de liderazgo deben ir unidas a la competencia de tomar riesgos considerados en enfermería por ejemplo, y por lo tanto en la salud, de lo contrario no se alcanzará todo el potencial para mejorar la atención médica de calidad que la gente necesita (20); adicionalmente conocer el contexto toma relevancia como competencia para los líderes de la salud, que apoyan la generación de empleados comprometidos con habilidades de liderazgo, dando lugar a mejores prácticas asistenciales (21).

En el ámbito de los servicios de salud, la concepción acerca del genero cobra relevancia al momento de generar procesos de desarrollo y aprendizaje, cada vez más el número de mujeres líderes se está expandiendo, las mujeres que desempeñan roles de liderazgo 
del área de la salud, debido a la creciente participación de las mujeres en pregrado y postgrado en programas de atención en salud, de los cuales las mujeres representan el 60 por ciento de las matrículas realizadas en dichos programas según el Censo de los EE.UU (22).

Mientras que en el sector salud, dos tercios de los médicos son hombres, el número de médicos de sexo femenino con licencia para ejercer también ha aumentado en los últimos dos años en un ocho por ciento en comparación con sólo el dos por ciento para los hombres. Los médicos de sexo femenino tienden a ser más jóvenes; 34 por ciento tienen 39 años o menos, en comparación con sólo el 18 por ciento de los médicos de sexo masculino (23). Por lo que es importante, tener en cuenta aspectos como los mencionados en los procesos de formación, desarrollo de competencias y satisfacción laboral, destacando el rol de las mujeres para que "ejerzan el liderazgo desde sus propias características, en vez de imitar el tradicional rol de liderazgo masculino"(24), en la espera de formar lideres sensibles a los problemas sociales que se presentan en el sector y que están relacionados con las prácticas de liderazgo(25).

A nivel de las instituciones de salud, se necesita que el personal clínico cuente con habilidades de liderazgo y trabajo en equipo para que puedan generar cambios estratégicos, tanto a nivel de la organización como a nivel interpersonal, habilidades necesarias para sostener la mejora del comportamiento y los cambios todas las personas involucradas en el cuidado de pacientes y clientes (25). Las estrategias claves de éxito son la educación y el establecimiento de oportunidades internas para difundir los resultados; la transformación del liderazgo del personal de enfermería impulsa el cambio organizacional y proporciona la visión, los recursos y el tiempo favoreciendo la gestión administrativa y financiera de la instituciones (26).

\section{IMPACTO DEL LIDERAZGO SOBRE EL TRABAJADOR DE LA SALUD}

El Impacto del liderazgo en los trabajadores de la salud, es de gran interés en la medida que impacta la satisfacción laboral y la calidad de la atención, un estudio analizó el estilo de liderazgo y el efecto en la comunicación supervisor-subordinado y el compromiso organizacional de la enfermería en la atención sanitaria, generando discusión alrededor del tema, ya que no es concluyente pero plantea que moderar la relación entre la 
comunicación entre el supervisor y subordinado, y determinados estilos de liderazgo pueden generar compromiso organizacional (27).

Las organizaciones del sector de la salud en el mundo están experimentando reformas estructurales, como en la mayoría de las reformas es relevante el compromiso de los empleados y el apoyo de los directivos de los hospitales, por lo que los administradores deben asumir retos, mediante un estudio que analizo la relación entre el liderazgo carismático que es el que comúnmente surge en momentos de estrés y crisis, y los comportamientos de los seguidores, encontró que la autonomía en el trabajo y el estrés en el lugar de trabajo unido al liderazgo carismático orienta mejoradas en los trabajadores. Los resultados de este estudio indican que el liderazgo carismático tiene una influencia positiva en el comportamiento orientado a la iniciativa (28-31)

Otro aspecto es la implementación en las organizaciones que prestan servicios de salud, en la formación para el desarrollo del líder en la medida que este impacta la calidad del servicio, las habilidades y competencias de los líderes en una organización en el contexto de la estructura organizacional deben garantizar una reserva de talento para el liderazgo, lo cual es clave en cargos enfocados a los logros con el objetivo de mejorar la calidad del servicio (32).

\section{LIDERAZGO Y CALIDAD EN EL SERVICIO DE SALUD}

Un servicio con calidad implica la generación de curvas de aprendizaje sostenidas en el tiempo, para lo cual es fundamental la retención del personal, para el caso del sector salud la retención del personal de enfermería es un problema importante que enfrenta la atención, agravada por las diferencias intergeneracionales en los valores de trabajo y las necesidades de fuerza de trabajo. El liderazgo efectivo, el trabajo en equipo y un seguimiento constructivo al personal de enfermería son factores importantes que influyen en las tasas de retención de enfermeras y ayudan a construir una cohesión en el lugar de trabajo. Los gerentes deben reconocer los retos y oportunidades asociados a la diversidad generacional y entender lo que las enfermeras de diferentes generaciones consideran como rasgos deseables de gestión. La clave para la retención es el desarrollo de políticas y prácticas que se centran en la generación de competencias, utilización de las fortalezas y habilidades que caracterizan a las distintas generaciones de las 
enfermeras y la creación de un entorno de trabajo en el que las enfermeras de todas las generaciones se sientan apoyados y valorados (32).

Lo anterior es un compromiso para los gerentes de recursos humanos, por ejemplo, un estudio muestra como los gerentes de recursos humanos que participaron en un programa de desarrollo de liderazgo una vez culminado concluyeron, que el impacto de los procesos de formación en liderazgo son significativos en la medida que marcan la diferencia, afirmando "habíamos hablado de los retos antes, pero el programa de liderazgo en realidad tiende a movilizarnos" (33).

La calidad percibida de un servicio hospitalario está relacionado con aspectos de motivación, satisfacción laboral, liderazgo, un estudio realizado hace varias décadas planteaba esta premisa como eje fundamental, en el Hospital "Mario Muñoz Monroy" determinó que la motivación laboral es la variable que mayor correlación tiene con la calidad, seguida por el liderazgo y la satisfacción laboral (34). Complementario a estos hallazgos, otro estudio evidencia relación directa entre el liderazgo y la satisfacción laboral a través de la motivación intrínseca.

En el ámbito de la salud, una de las profesiones en las que se ha estudiado ampliamente el impacto de las habilidades de liderazgo en la satisfacción laboral y la calidad de la atención, es la enfermería, en la que el liderazgo transformacional ejercido por los gerentes de servicios de enfermería tiene un gran efecto sobre el nivel de satisfacción laboral de las enfermeras clínicas y sugiere que es esencial para proporcionar programas de capacitación en habilidades de liderazgo para mejorar el conocimiento de las enfermeras gestoras, además de la supervisión, las enfermeras gestoras deben motivar a las enfermeras clínicas para llevar a cabo el trabajo de enfermería clínica con entusiasmo (35) . Las enfermeras tienden a estar más satisfechas con el liderazgo transformacional que con el estilo de liderazgo transaccional, por lo tanto, los gestores de personal de enfermería deben usar el estilo de liderazgo transformacional con el fin de aumentar la satisfacción en el trabajo (36).

El efecto relativamente más fuerte del liderazgo transformacional está sobre la satisfacción laboral, sobre todo en los servicios con baja estabilidad laboral, se sugiere la importancia de la utilización de diferentes comportamientos de liderazgo para 
garantizar la satisfacción laboral entre el personal con el fin tener una alta estabilidad laboral (37).

El impacto de los estilos de liderazgo en la satisfacción del enfermero se ve reflejado en la estabilidad laboral y la intensión de permanecer en la institución en la que se desempeña, un estudio que tuvo como objetivo examinar el impacto de los estilos de liderazgo de los gerentes de enfermería en la satisfacción laboral, analizó que el aumento de satisfacción de las enfermeras es uno de los elementos clave para afrontar los retos de los resultados de calidad, satisfacción del paciente y la retención del personal de enfermería en los hospitales, dicha satisfacción pone en evidencia la necesidad de una mayor atención a la formación y el desarrollo de comportamientos de liderazgo efectivo (38).

\section{RESULTADOS}

El presente artículo ofrece un punto de partida para el desarrollo de hipótesis acerca de cómo el liderazgo en los servicios de salud, favorece los resultados en la gestión por procesos para mejorar la calidad y disminuir los costos y a su vez juega un papel fundamental en la gestión de los recursos humanos favoreciendo la satisfacción laboral que se ve reflejada en la calidad de la atención.

Los trabajadores de la salud, por lo general están a la espera de identificar problemas y resolverlos aportando a la mejora de los procesos y los resultados de la gestión diaria, y a su vez detallan comportamientos de liderazgo necesarios para una organización exitosa (39). Es así como el presente artículo plantea dos hipótesis complementarias, una el liderazgo en los servicios de salud, implica comprender el liderazgo como un componente fundamental del proceso de gestión y administración y dos el liderazgo como competencia de los trabajadores en los servicios de salud impacta la satisfacción laboral y la calidad de la atención.

El liderazgo en los servicios de salud, es de gran importancia en el sector en la medida que favorece la creación de estructuras y procesos para favorecer la sostenibilidad de 
las instituciones, por lo que un programa innovador en formación de habilidades de liderazgo construye dirigentes excepcionales.

En los servicios de salud, la autogestión, la planificación, control y capacidad de liderazgo en relación con su capacidad para ofrecer servicios de salud, sugiere que a pesar que los directivos son capaces de planificar y prever el camino a seguir y crear un entorno favorable para ellos y su personal, la falta de capacidad de ejecución debe conducir en el futuro desarrollo de la gestión y la formación en programas dirigidos a las gestoras de personal de enfermería por ejemplo en temas de habilidades de liderazgo unido a habilidades de gestión para favorecer la planificación estratégica y la toma de decisiones efectivas como competencia fundamental de los líderes de procesos (40).

Es importante resaltar como los departamentos de educación de los hospitales pueden articularse con la dirección estratégica de la organización, al priorizar temas relacionados con la gestión, la eficacia de los procesos de trabajo, y centrarse en lo que más importa a la organización cuando la organización adopta un proceso de evaluación de excelencia y desempeño. Los procesos sistemáticos deben integrar las mejores prácticas de gestión de organizaciones para lograr mejores resultados, por ejemplo organizaciones de atención en salud de alto rendimiento que han obtenido premios nacionales, con ejemplos de cómo los departamentos de educación de las organizaciones de alto rendimiento reajustado sus planes de educación más proactivos, centrado en los resultados y generación de habilidades de liderazgo (41) .

Más allá del liderazgo como componente fundamental de la gestión de las instituciones de salud, se debe analizar el liderazgo como competencia del personal asistencial para favorecer la satisfacción laboral. Los programas tendentes a la gestión del cambio desarrollados por personal de enfermería, sugieren que los supervisores opuestos a estilos de liderazgo de tipo jerárquico y las remuneraciones que premian la falta de ausencias también pueden conseguir disminuciones en indicadores de seguimiento al personal como el absentismo, el cual puede considerarse como un resultado final y una consecuencia del grado de satisfacción de los profesionales (42) .

Estudios relacionan el perfil de liderazgo de los gerentes de los departamentos de enfermería con la satisfacción laboral del personal. Los buenos gerentes de enfermería 
desempeñan un papel importante en la retención y la satisfacción del personal, a su vez mejorando la retención se conduce a un ahorro para la empresa, generando recursos que pueden ser asignados a actividades tales como la capacitación y orientación para ayudar a las enfermeras líderes en el desarrollo de estas habilidades (43).

Experiencias positivas del personal de dirección son fundamentales para garantizar la satisfacción en el trabajo y la retención de la fuerza laboral, la prestación de servicios con calidad y el bienestar de los beneficiarios de los servicios. Los atributos esenciales de un buen liderazgo de los mandos intermedios favorecen la construcción de una cultura organizacional con respeto, el valor del reconocimiento y el trabajo en equipo, así como la comunicación efectiva y la flexibilidad. Sin embargo, el éxito del liderazgo y los resultados de la gestión dependen de una buena organización de los procesos de liderazgo y la formación de los lideres, ya que no existe una preparación adecuada para ocupar cargos directivos de mandos medios en el sector y la falta de una clara directriz y estandarización de indicadores clave de rendimiento para evaluar las habilidades de liderazgo y gestión (44).

\section{CONCLUSIONES}

La anterior revisión respecto al liderazgo en los servicios de salud muestra la importancia de estudiar el efecto transformador del liderazgo a nivel de las instituciones en pro del bienestar mismo de los trabajadores para contribuir al logro de la misión de las instituciones de salud y contribuir a establecer prioridades estratégicas para la gestión exitosa de las instituciones.

El reto para los servicios de salud está en ampliar el conocimiento y potenciar el liderazgo como factor clave de la gestión para la prestación de los servicios de salud con alto estándares de calidad, unido lo anterior a la planeación estratégica, el liderazgo es un 
componente fundamental de la gestión directiva y administrativa que va más allá de ser un tema de interés empresarial desde la perspectiva de los altos cargos directivos, gerenciales y el personal operativo.

Por otra parte, el Impacto del liderazgo en los trabajadores de la salud, es de gran interés organizacional en la medida que impacta la satisfacción laboral y la calidad de la atención. Otro aspecto a resaltar, es la implementación de procesos de educación y desarrollo de competencias en las organizaciones que prestan servicios de salud, que contribuyan a la formación para el desarrollo del líder como generador de una atención con calidad; adicionalmente, las habilidades y competencias de los líderes en una organización en el contexto de la estructura organizacional deben garantizar una reserva de talento humano para el liderazgo, lo cual es clave en cargos enfocados a los logros con el objetivo de mejorar la calidad del servicio, ya que el impacto de los estilos de liderazgo en la satisfacción del personal asistencial se ve reflejado en la estabilidad y satisfacción laboral.

Finalmente, la competencia de liderazgo es fundamental en la gestión de procesos, prestación de servicios con calidad y en la generación de trabajadores laboralmente satisfechos. Es importante resaltar el papel de los departamentos de educación de los hospitales los cuales al articularse con la dirección estratégica de la organización, y al priorizar temas relacionados con la gestión, la eficacia de los procesos de trabajo, se centran en programas de formación en habilidades de liderazgo para favorecer mejores resultados institucionales.

\section{AGRADECIMIENTOS}

Universidad del Rosario y Méderi. Financiado en el marco del Doctorado de Ciencias de la Dirección 2013.

\section{BIBLIOGRAFÍA}

1. Sotelo J. World's public health today. Universidad Peruana Cayetano Heredia [Internet]. 2013.

2. Mintzberg $\mathrm{H}$. Managing the myths of health care. World Hospitals and Health Services. University M, editor2012. 
https://doi.org/10.22519/21455333.413

3. Garratt A. National and cross-national surveys of patient experiences: a structured review. OECD [Internet]. 2008. Available from: www.oecd.org/dataoecd/43/58/39493930.pdf.

4. Castner J. A Leadership Challenge. The Journal of Noursing Administration. 2012:46772.

5. $\quad$ Curry L. Experiences of leadership in health care. Human Resources for Health. 2012:2-

8.

6. Ingebrigtsen T, Georgiou A, Clay-Williams R, Magrabi F, Hordern A, Prgomet M, et al. The impact of clinical leadership on health information technology adoption: Systematic review. International Journal of Medical Informatics. 2014 6//;83(6):393-405.

7. O'Neil E, Chow MP. Leadership Action for a New American Health System. Nurse Leader. 2011 12//;9(6):34-7.

8. Menaker R. Leadership Strategies in Healthcare. Medical Practice Management. 2009:339-43.

9. Carey W. Coaching models for leadership development: an integrativereview. Journal of leadership studies. 2011:51-69.

10. Tlotlego N, Nonvignon J, Sambo L, Asbu E, Kirigia J. Assessment of productivity of hospitals in Botswana: a DEA application. Int Arch Med. 2010;3(27).

11. Fitzgerald L, Ferlie E, McGivern G, Buchanan D. Distributed leadership patterns and service improvement: Evidence and argument from English healthcare. The Leadership Quarterly. 2013 2//;24(1):227-39.

12. Hewison A, Morrell K. Leadership development in the English National Health Service: A counter narrative to inform policy. International Journal of Nursing Studies. 2014 4//;51(4):67788.

13. Parry K, Mumford MD, Bower I, Watts LL. Qualitative and historiometric methods in leadership research: A review of the first 25\&\#xa0;years of The Leadership Quarterly. The Leadership Quarterly. 2014 2//;25(1):132-51.

14. Jeon $\mathrm{YH}$, Merlyn T, Chenoweth L. Leadership and management in the aged care sector: a narrative synthesis. Australasian journal on ageing. 2010;29(2):54-60.

15. Briggs D, Smyth A, Anderson J. In Search of Capable Health Managers: what is distinctive about health management and why does it matter? Asia Pacific Journal of Health Management. 2012;7(2).

16. Clark J, Spurgeon P, Hamilton P. Medical professionalism: leadership competency-an essential ingredient. The International Journal of Clinical Leadership. 2008;16(1):3-9.

17. TOUSSAINT J. A Management, Leadership, and Board Road Map to Transforming Care for Patients. Frontiers of health services management. 2013;29(3):3-15.

18. Calhoun JG, Dollett L, Sinioris ME, Wainio JA, Butler PW, Griffith JR, et al. Development of an interprofessional competency model for healthcare leadership. Journal of Healthcare Management. 2008;53(6).

19. Lorinkova NM, Pearsall MJ, Sims HP. Examining the differential longitudinal performance of directive versus empowering leadership in teams. Academy of Management Journal. 2013;56(2):573-96.

20. Chesbrough HW. Open innovation: The new imperative for creating and profiting from technology: Harvard Business Press; 2003.

21. Wikström E, Dellve L. Contemporary leadership in healthcare organizations: fragmented or concurrent leadership. Journal of health organization and management. 2009;23(4):411-28.

22. Price A, Howard D. Connect for success: social leadership, mentorship, and the female healthcare executive. Front Health Serv Manage. 2012;28(4):33-8.

23. Angood P. Demographics, Shifting ModeLs of Care and Physician Leadership. Physician Executive. 2013;39(3):4.

24. Contreras Torres F, Pedraza Ortiz JE, Mejía Restrepo X. La mujer y el liderazgo empresarial. Diversitas. 2012;8(1):183-93. 
25. Kendrick M. Advocacy as Social Leadership. International Journal of Leadership in Public Services, The. 2008;4(3):62-70.

26. Hauck S, Winsett RP, Kuric J. Leadership facilitation strategies to establish evidence based practice in an acute care hospital. Journal of advanced nursing. 2013;69(3):664-74.

27. Işik O, Uğurluoğlu Ö, Akbolat M, Öner ZH, Pisapia J. Charismatic leadership: impulse factor for initiative-oriented health care personnel in the Turkish public hospitals. HealthMED. 2012;6(5).

28. Weinberg R, McDermott M. A comparative analysis of sport and business organizations: Factors perceived critical for organizational success. Journal of Applied Sport Psychology. 2002;14(4):282-98.

29. Goldsmith M, Lyons L, Freas A. Coaching for leadership: How the world's greatest coaches help leaders learn: Jossey Bass/Pfeiffer; 2000.

30. Henochowicz S, Hetherington D. Leadership coaching in health care. Leadership \& Organization Development Journal. 2006;27(3):183-9.

31. Taylor CA, Taylor JC, Stoller JK. The influence of mentorship and role modeling on developing physician-leaders: Views of aspiring and established physician-leaders. Journal of general internal medicine. 2009;24(10):1130-4.

32. Nelsey L, Brownie S. Effective leadership, teamwork and mentoring-Essential elements in promoting generational cohesion in the nursing workforce and retaining nurses. Collegian: Journal of the Royal College of Nursing Australia. 2012;19(4):197-202.

33. O'Neil ML. Human resource leadership: the key to improved results in health. Human resources for health. 2008;6(1):10.

34. Hernández Junco V, Quintana Tápanes L, Mederos Torres R, Guedes Díaz R, García Gutiérrez BN. Motivación, satisfacción laboral, liderazgo y su relación con la calidad del servicio. Revista Cubana de Medicina Militar. 2009;38(1):0-.

35. Wang X, Chontawan R, Nantsupawat R. Transformational leadership: effect on the job satisfaction of registered nurses in a hospital in China. Journal of advanced nursing. 2012;68(2):444-51.

36. Negussie N, Demissie A. Relationship between leadership styles of Nurese managers and nurses' job satisfaction in Jimma University Specialized Hospital. Ethiopian journal of health sciences. 2013;23(1):50-8.

37. Havig AK, Skogstad A, Veenstra M, Romøren TI. The effects of leadership and ward factors on job satisfaction in nursing homes: a multilevel approach. Journal of clinical nursing. 2011;20(23 24):3532-42.

38. Abualrub RF, Alghamdi MG. The impact of leadership styles on nurses' satisfaction and intention to stay among Saudi nurses. Journal of nursing management. 2012;20(5):668-78.

39. Toussaint J. A Management, Leadership, and Board Road Map to Transforming Care for Patients. Frontiers Of Health Services Management. 2013:2-15.

40. Pillay R. The skills gap in nursing management in the South African public health sector. Public Health Nursing. $2011 ; 28(2): 176-85$.

41. Burke KM, Hellwig SD. Education in high-performing hospitals: using the Baldrige framework to demonstrate positive outcomes. Journal of continuing education in nursing. 2011;42(7):299-305; quiz 6-7, 36.

42. Blanca-Gutiérrez JJ, Jiménez-Díaz MdC, Escalera-Franco LF. Intervenciones eficaces para reducir el absentismo del personal de enfermería hospitalario. Gaceta Sanitaria. 2013;27(6):545-51.

43. Duffield CM, Roche MA, Blay N, Stasa H. Nursing unit managers, staff retention and the work environment. Journal of clinical nursing. 2011;20(1 2):23-33. 
https://doi.org/10.22519/21455333.413

44. Jeon Y-H, Glasgow NJ, Merlyn T, Sansoni E. Policy options to improve leadership of middle managers in the Australian residential aged care setting: a narrative synthesis. BMC Health Services Research. 2010;10(1):190. 